\title{
Perinatal health care providers' approaches to recommending and providing pertussis vaccination in pregnancy: a qualitative study
}

\author{
Hana Mijović MD MSc, Devon Greyson PhD MLIS, Emily Gemmell MPH, Marie-Ève Trottier MSc, \\ Maryline Vivion PhD, Janice E. Graham PhD, Ève Dubé PhD, Julie A. Bettinger PhD MPH
}

\section{Abstract}

Background: In 2018, the Canadian National Advisory Committee on Immunization and the Society of Obstetricians and Gynaecologists of Canada recommended a single dose of tetanus toxoid, reduced diphtheria toxoid and reduced acellular pertussis (Tdap) vaccine in every pregnancy. To understand how perinatal health care providers in Canada are translating recent recommendations for universal antenatal Tdap vaccine into routine clinical practice, we examined health care providers' perceptions of what influences their ability to recommend and provide Tdap vaccine consistently to pregnant women.

Methods: Between June 2018 and July 2019, we conducted semistructured telephone interviews with perinatal health care providers (nurses, midwives, family physicians and obstetricians) from 5 provinces (British Columbia, Manitoba, Ontario, Quebec and Nova Scotia) representing diverse educational experiences, practice settings and models of care. We analyzed the data using interpretive description.

Results: We interviewed 44 perinatal health care providers (13 family physicians, 12 midwives, 10 obstetricians and 9 nurses) practising in a variety of settings. Health care providers' ability to recommend and provide antenatal Tdap vaccine was strongly influenced by structural constraints in the Canadian perinatal health care system. The participants' clinical training varied, which resulted in different knowledge and practices. Participants felt hindered by a lack of lay information resources. Consistent and convenient vaccine access was perceived to be key to promoting confidence and encouraging uptake, yet antenatal Tdap vaccine was not easily accessible for all women.

Interpretation: Our findings suggest that Canada's fragmented health care model has a detrimental effect on health care providers' ability to recommend and ensure access to antenatal Tdap vaccine. Lessons from this study are pertinent to the implementation of successful pertussis vaccine programs and future pregnancy vaccination initiatives.

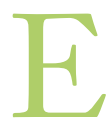
ndemic pertussis contributes to childhood morbidity and mortality in Canada, particularly among infants younger than 4 months of age..$^{1,2}$ A single dose of tetanus toxoid, reduced diphtheria toxoid and reduced acellular pertussis (Tdap) vaccine during pregnancy boosts maternal pertussis antibodies and provides passive protection for newborn infants until they are old enough to be vaccinated. ${ }^{3}$ In 2018, the National Advisory Committee on Immunization (NACI) recommended Tdap vaccination in every pregnant woman between 27 and 32 weeks' gestation, and the Society of Obstetricians and Gynaecologists of Canada (SOGC) recommended it between 21 and 32 weeks. ${ }^{2,4}$ The vaccine is publicly funded for every pregnancy in all Canadian provinces and territories except British Columbia and Ontario. ${ }^{5}$ This was the second vaccine to be recommended routinely in pregnancy in Canada since influenza vaccine, in 2007. New vaccines to be administered during pregnancy are under development and may be recommended routinely in the future; ${ }^{6}$ therefore, an understanding of perinatal vaccine counselling and provision is important.

Evidence-based vaccine recommendations by professional bodies are effective only when they can be translated consistently into clinical practice. Canadian family physicians, midwives, nurses and obstetricians all provide, and frequently share, care for pregnant women. Given the diversity of educational experiences, practice settings and models of care among

Competing interests: None declared.

This article has been peer reviewed.

Correspondence to: Julie Bettinger, jbettinger@bcchr.ubc.ca CMAJ Open 2020. DOI:10.9778/cmajo.20190215 
the perinatal health care provider workforce, the ability to recommend and provide vaccines in pregnancy may vary. ${ }^{7-9}$

Health care provider recommendation is a well-established determinant of vaccine acceptance and uptake in pregnant women. ${ }^{10-14}$ Studies from other high-income countries with universal antenatal Tdap programs show that pregnant women are generally open to receiving Tdap but that vaccine uptake remains suboptimal because health care providers do not recommend the vaccine consistently and because Tdap often is not conveniently accessible through comprehensive perinatal care. ${ }^{15-19}$

We aimed to understand how perinatal health care providers in Canada are translating recent NACI and SOGC recommendations for universal antenatal Tdap vaccination into routine clinical practice. We examined health care providers' perceptions of what influences their ability to recommend and provide antenatal Tdap vaccine consistently to pregnant women in 5 Canadian provinces.

\section{Methods}

\section{Setting and recruitment}

We aimed to generate a high-level understanding of the implementation of Canada's national recommendation for Tdap in every pregnancy. We recruited health care providers from BC, Manitoba, Ontario, Quebec and Nova Scotia. Together these 5 provinces constitute about $80 \%$ of the Canadian population. ${ }^{20}$

We purposively recruited participants meeting the following eligibility criteria: obstetrician-gynecologist, family physician, general practitioner, registered nurse, nurse practitioner or registered midwife currently providing care to pregnant women in the 5 provinces. We identified lists of potential participants in each province with the assistance of disciplineand province-specific collaborators. Invitations were sent via email or ground mail on a rolling basis to collect a maximally diverse sample with regard to clinical discipline, practice setting (urban/suburban/rural), province and population served (including the general population, patients at high and low risk medically, Indigenous patients and patients of low socioeconomic status). Participants provided online consent, and eligibility criteria were reviewed before the interview to confirm eligibility. Recruitment ended when new themes were no longer being identified in the interviews and we were no longer adding meaningful diversity to the study population. All participants provided informed consent.

\section{Data collection}

All participants completed a demographic questionnaire online in response to the study invitation, before the interview was scheduled or at the time of the interview. Telephone or in-person interviews were conducted from June 2018 to July 2019 in English or French (depending on the participant's preference) with the use of a semistructured interview guide that was developed based on a literature review, the researchers' previous vaccination research ${ }^{12,21-24}$ and the sensitizing concepts of the study (Appendix 1, available at www.cmajo-
pen.ca/content/8/2/E377/suppl/DC1). The guide was translated from English into French by 2 team members fluent in both languages (È.D., M.V.). The interview guide was pilottested with 3 clinicians at BC Children's Hospital who were not study participants.

The interviews lasted about 30 minutes and were conducted by a female graduate or postgraduate qualitative health researcher (D.G., E.G., H.M., M.-E.T., M.V.). Interviews explored the health care provider's training and clinical practice setting, how he or she learned about and implemented clinical guidelines, experience with vaccines in pregnancy and approaches taken with vaccine-hesitant patients.

In keeping with the qualitative principle of emergent design, ${ }^{25}$ data collection and analysis were iterative, with coding of initial interviews beginning before all data were collected. This permitted adjustment of questions and verification of findings emerging from early data collection in subsequent interviews. For example, several study participants expressed being unclear about the new NACI and SOGC Tdap recommendations. We therefore explored this issue further by specifically asking subsequent participants how they learned about the Tdap recommendations and what additional information (if any) they needed.

Interviews were audio-recorded and transcribed in the language used. Participants were invited to review final, deidentified transcripts for accuracy (no changes were requested).

\section{Data analysis}

We coded the data using a 2-stage emic-etic approach. ${ }^{26}$ Transcripts were first coded deductively in their original language with NVivo Software (QSR International) to identify passages relevant to the research question (H.M., M.-E.T., M.V.). Inductive coding was done on selected passages (H.M., with verification by M.-E.T. and M.V. for French-language transcripts) to explore relations among and within the data categories identified through deductive coding. The entire study team then proceeded with inductive analysis informed by interpretive description, a qualitative analytic approach used to expand on existing knowledge and provide new, clinically applicable insights. ${ }^{27}$ Participants did not provide input into the analysis.

\section{Ethics approval}

This study received approval from the research ethics boards of the IWK Health Centre, the Centre de recherche du Centre hospitalier universitaire de Québec-Université Laval and the University of British Columbia.

\section{Results}

We sent 212 study invitations and received replies from 58 health care providers, of whom 52 consented and 6 declined. Of the 52 providers who consented, 6 were not available for an interview, and 2 were excluded because they did not meet eligibility criteria. We thus interviewed 44 eligible health care providers practising in a variety of settings (Table 1). 
Table 1: Study participants' characteristics

\begin{tabular}{|c|c|}
\hline Characteristic & $\begin{array}{c}\text { No. }(\%) \text { of } \\
\text { participants } \\
n=44\end{array}$ \\
\hline \multicolumn{2}{|l|}{ Professional affiliation } \\
\hline Family physician & $13(30)$ \\
\hline Midwife & $12(27)$ \\
\hline Nurse & $9(20)$ \\
\hline Obstetrician & $10(23)$ \\
\hline \multicolumn{2}{|l|}{ Province of practice } \\
\hline British Columbia & $13(30)$ \\
\hline Manitoba & $7(16)$ \\
\hline Nova Scotia & $5(11)$ \\
\hline Ontario & $8(18)$ \\
\hline Quebec & $11(25)$ \\
\hline \multicolumn{2}{|l|}{ Community of practice } \\
\hline Rural (< 1000 inhabitants) & $3(7)$ \\
\hline Small town (1000 to < 30000 inhabitants) & $11(25)$ \\
\hline $\begin{array}{l}\text { Medium town }(30000 \text { to }<100000 \\
\text { inhabitants) }\end{array}$ & $3(7)$ \\
\hline Urban centre ( $\geq 100000$ inhabitants) & $27(61)$ \\
\hline \multicolumn{2}{|l|}{ Years in practice } \\
\hline Mean (range) & $13(1-43)$ \\
\hline Median & 12 \\
\hline $\begin{array}{l}\text { Provided antenatal Tdap vaccine at point of } \\
\text { care }\end{array}$ & $18(41)$ \\
\hline \multicolumn{2}{|c|}{$\begin{array}{l}\text { Note: Tdap = tetanus toxoid, reduced diphtheria toxoid and reduced acellular } \\
\text { pertussis. } \\
\text { "Except where noted otherwise. }\end{array}$} \\
\hline
\end{tabular}

Participants described a trusting relationship between a provider and his or her pregnant patient as the foundation for vaccine discussions (Box 1). However, health care providers' ability to recommend and provide Tdap vaccine consistently was shaped by factors that were often beyond their control (Box 2). These health care system barriers included clinical training opportunities, availability of appropriate information for patients (Box 3 ) and patient access to vaccination (Box 4).

\section{Patient-provider relationship}

Health care providers said most of their patients were not aware of the new Tdap recommendations. Patient acceptance of Tdap was strongly influenced by their trust in their health care provider's recommendation (Box 1). Physicians and nurses reported leveraging their rapport to make an unequivocal recommendation for Tdap vaccine. However, midwives' approach to vaccine counselling varied: some described recommending Tdap vaccine and following up on their client's vaccination status in subsequent visits, whereas others said expressing a personal opinion or making a recommendation in favour of vaccine could compromise the principle of informed choice that is essential to the Canadian midwifery model of
Box 1: Examples of quotes reflecting participants' views on the patient-provider relationship

- They're not just trusting the science, they're trusting the person delivering the science. (Family physician, urban centre, British Columbia)

- It comes down to having a good relationship with people, and having that trust over time and being in the community for [many] years now and knowing all these families for such a long time, following them through their pregnancies, seeing them with other kids. (Family physician, Nova Scotia*)

- I think they've established quite a bit of trust with me, and if they're people who might be a bit skeptical about some of the advice their doctor gives them, I think they might be more open to hearing it [vaccine recommendations] from a midwife, who they might perceive as being more sympathetic to their questions or concerns about vaccines. (Midwife, urban centre, Ontario)

- I try not to sway [clients] in any way. I just try to give them information on [vaccines] and what is recommended at this time. (Midwife, small town, Manitoba)

- I can't be good at everything ... so I refer to public health because they are the ones that keep up to date on [vaccine recommendations], and they are the ones that are always given the new information. (Midwife, medium-size town, Manitoba)

${ }^{*}$ Community setting not specified in order to protect participant anonymity in a small province.

Box 2: Examples of quotes reflecting participants' views on clinical training

- We would like to have access to the latest, evidence-based information. We can't just say to women "This is the last recommendation," we need to be able to give them the evidence. ... Having actual numbers to quote would help.* (Midwife, urban centre, Quebec)

- I think part of ... [the value of clinical training] was that all of us [midwives] were together. ... It is useful to hear what your peers and colleagues think and have the opportunity to ask questions. (Midwife, Nova Scotia*)

- [I may prioritize vaccine training] if it was focused on very short, very useful things. There's just so much else that's in the queue for [continuing medical education]. (Family physician, small town, British Columbia)

- I think recommending vaccines in pregnancy can be really challenging because there is a lot of vaccine hesitancy among pregnant patients. And so, if you don't feel really well prepared with information, and not just the information but actually how to talk to people about it, then getting any pushback can be very challenging ... creating a difficult relationship between you and your patient. (Family physician, urban centre, Manitoba)

*Translated from French.

†Community setting not specified in order to protect participant anonymity in a small province.

care. They saw their role as informing women about the Tdap vaccine recommendations by the NACI and SOGC, and then directing them to public health or physicians for further advice and vaccine counselling.

\section{Clinical training}

Although all participants were aware of the Tdap vaccine recommendations, their practice setting influenced both vaccine 
Box 3: Examples of quotes reflecting participants' views on lay information resources

- Having something that ... provides [patients] with the information they care about, like "Why now, Canada? And what are the risks, what do we know safety wise, and what are the expected benefits?" would just help. ... It's not that l'm going to not say those things, but it's good for me to say them and then have them take a small part home. (Obstetrician, urban centre, Ontario)

- The [Society of Obstetricians and Gynaecologists of Canada] pamphlet is a bit complex in terms of describing placental transfer. So I break that down with patients a little bit better so they understand that it's not really for them, that it's for baby. (Nurse, urban centre, Ontario)

- Instead of saying the Internet is bad and "Dr. Google" is bad, we need to recognize that it's a tool. ... Let's actually use the technology of the day [to provide information to patients]. But that actually requires funding and a body of work, and whose job is it to do that? (Family physician, urban centre, British Columbia)

\section{Box 4: Examples of quotes reflecting participants' views on} vaccine access

- The recommendation for Tdap was made before ensuring that infrastructure was in place to provide it.* (Obstetrician, urban centre, Quebec)

- There's this sort of doubt about being what is perceived to be the guinea pig. Well, if it was really indicated, the government would cover it. (Family physician, urban centre, British Columbia)

- I work at a very technology-depressed area, and most people cannot pay for things that aren't covered. ... I generally haven't been discussing the pertussis shot with people. (Family physician, rural community, $\mathrm{BC}$ )

- We have the good fortune of being in a family medicine clinic, and so there's a vaccine fridge. ... Having the flu shot is just a given, having the pertussis booster is a given. I didn't have to do anything to make that happen. ... So whereas if you're in a clinic that's only [obstetrician/gynecologists], or if it's all specialists, they may not have the facilities to do that. (Obstetrician, small town, Ontario)

- If I told [patients] to go somewhere else, they would never go. They don't even show up to most of their appointments with me. So if we don't do something at the moment that we have that window, it doesn't get done with people who don't have a car and don't have a licence, and if they're using substances, they may not be able to keep good track of time. (Family physician, urban centre, Manitoba)

Note: Tdap = tetanus toxoid, reduced diphtheria toxoid and reduced acellula pertussis.

${ }^{*}$ Translated from French

knowledge acquisition and their ability to incorporate vaccine recommendations consistently into their clinical work (Box 2). Participant descriptions of how they learned about and implemented the Tdap vaccine recommendation in their practice showed that the NACI and SOGC recommendations were not disseminated via coordinated training of health care providers. Providers who drew on their existing experience providing childhood vaccines or other vaccines during pregnancy (e.g., nurses, urban family physicians) felt well prepared to recommend and provide Tdap vaccine. Rural family physicians and midwives, working in jurisdictions where vaccines were delivered by public health, described feeling less prepared to discuss Tdap vaccination. With multiple, competing priorities for continuing medical education, vaccine-related training was perceived as less directly relevant to clinical practice for these providers.

When asked for suggestions about vaccine continuing education, providers agreed such training should be succinct, equipping them with patient-directed information and practical suggestions on how best to communicate the information in their practice settings. This included the rationale for the new vaccine recommendations, risk of infant pertussis, vaccine effectiveness, approaches to vaccine-hesitant patients, and clarification about vaccine funding and access. Some providers valued interactive training sessions that facilitated discussion. Many participants also emphasized the importance of being connected to academic institutions and of sharing vaccine updates or questions with colleagues through practice group meetings, academic rounds or online forums.

\section{Lay information resources}

Participants indicated that information resources for patients and their families were an important part of the vaccine discussion (Box 3). They felt hindered by a lack of appropriate, widely available resources for pregnant women. Participants reported that standardized paper and online resources, similar to those for childhood vaccines, would enable patients to verify information outside of the clinic visit and help validate health care providers' recommendations.

\section{Vaccine access}

Participants thought that convenient access to publicly funded vaccines was essential to enable vaccine uptake (Box 4). In an ideal scenario for vaccine uptake, a provider's vaccine recommendation would be followed by offering the vaccine immediately at the point of care, especially for patients who have difficulty navigating multiple medical appointments.

Participants with a vaccine refrigerator were able to vaccinate at the point of care. Providers who did not vaccinate were often frustrated when vaccine was not readily available through public health clinics or pharmacies. Some believed that the national recommendation for Tdap vaccine by the NACI and SOGC was made before adequate infrastructure was in place to provide the vaccine. Some participants, including but not limited to midwives, pointed out that vaccinating was not within midwives' scope of practice in Ontario and Quebec. Some wondered whether this might have a negative impact on vaccine uptake among midwifery clients.

Finally, health care providers in BC and Ontario were concerned that a lack of public funding for the vaccine in their provinces contributed to inequitable vaccine access for marginalized women. One provider reported not discussing Tdap vaccination at all because they thought recommending the vaccine put undue pressure on women without financial means to afford it. In addition, lack of public funding resulted 
in providers' having to counter patients' perceptions that antenatal Tdap vaccination was less important or less safe than publicly funded vaccines.

\section{Interpretation}

We identified major influences on perinatal health care providers' ability to provide and recommend antenatal Tdap vaccine consistently, as recommended by the NACI and SOGC. ${ }^{2,4}$ These included appropriate clinical training, lay information resources and vaccine access for patients.

Consistent with previous Tdap vaccine research done among pregnant women, ${ }^{15-19,28}$ our participants reported that their patients valued a confident vaccine recommendation by a trusted provider. It is common for patients to consult a range of sources about health information; ${ }^{22}$ therefore, widely available lay information resources for pregnant women may serve to reinforce the trust in and acceptance of health care provider recommendations. ${ }^{16}$ Our study highlights the variability in midwives' perceived role in vaccination, according to individual midwives' approaches to providing the informedchoice model of care ${ }^{29}$ and vaccine-related training..$^{21,30}$ The fact that providing vaccines to pregnant women is currently not within midwives' scope of practice in all provinces may also contribute to this variability. Routine recommendation and provision of vaccination by midwives could improve vaccine access and uptake in pregnancy. ${ }^{30,31}$

To be adequately prepared to recommend and provide vaccines, perinatal health care providers need current, consistent and reliable vaccine knowledge and access to concise training updates, as well as confidence in their communication skills and the time and ability to incorporate vaccine discussions into regular practice. ${ }^{32}$ Coordinated clinical training around antenatal Tdap vaccination for Canadian perinatal providers would ensure all health care providers have access to the same information and resources, improve vaccine communication skills and provide a chance for interdisciplinary collaboration. As suggested by our study participants, such training should be succinct, practice-focused and interactive. Linking vaccine updates with other educational activities could increase appeal to health care providers with multiple competing priorities. This coordinated training could also be implemented for other new vaccine recommendations.

Tdap vaccine is currently not accessible to all pregnant women in Canada. Vaccinating at point of care facilitates access but may not be realistic in all practice settings, as observed in Quebec. ${ }^{8}$ This finding underscores the need for ongoing communication and coordination between perinatal health care providers and public health units or pharmacies to optimize convenient access. Finally, lack of public funding in $\mathrm{BC}$ and Ontario further compromises vaccine access for women who cannot afford it.

A systems approach to delivery of vaccination programs is important to achieve high and inclusive vaccine uptake and to close the gap between national vaccine recommendations and clinical practice. ${ }^{11,33,34}$ In the Canadian context, this means implementing a coordinated, overarching nationwide pertussis vaccination program, ensuring the vaccine is publicly funded and easily accessible for all pregnancies in every province and territory, and ensuring that all perinatal providers are supported and trained appropriately and feel confident recommending the vaccine. Addressing potential gaps identified between the national-level vaccine recommendation and clinical practice in diverse health care settings may result in a more equitable and comprehensive pregnancy vaccination program.

Intervention research is required to support perinatal health care providers effectively in implementing clinical guidelines in their practice settings. The SOGC recently released an online course on vaccination in pregnancy for providers $^{35}$ along with a video and an e-book for patients. Evaluation of these resources will be helpful in determining whether the need for lay resources and clinical training identified in our study is being met. Given the variety of health care providers providing perinatal care, studies assessing initiatives to increase vaccine acceptance and access through interprofessional collaboration and integrated provision of care would be timely. This should include a nuanced discussion about ways to foster vaccine uptake while respecting women's autonomy in diverse communities and practice settings.

\section{Limitations}

Providers who agreed to participate in this study may have had greater vaccine knowledge and confidence than the typical Canadian perinatal health care provider. Nevertheless, many participants perceived that they could improve their vaccine knowledge and counselling skills, and desired clinically relevant training.

Owing to the lack of comprehensive pregnancy vaccination registries, we were unable to explore relations among the various barriers and facilitators identified by our participants and the vaccine uptake rates in their communities. With interviews taking place over 14 months, some of the earlier concerns raised by health care providers may have been resolved in some health jurisdictions, but the findings from our research are applicable with any new vaccine recommendation.

This study aimed to provide an overview of factors that influence Canadian health care providers' ability to recommend and provide Tdap vaccine and is not generalizable to every health care provider in every jurisdiction. Local studies would be able to further elicit jurisdiction-specific influences and inform local initiatives.

\section{Conclusion}

We identified major facilitators of and barriers to perinatal health care providers' ability to recommend and provide antenatal Tdap vaccine. The barriers highlighted by participants suggest that Canada's fragmented health care model has a detrimental effect on providers' ability to recommend and provide Tdap vaccine and ensure universal access in pregnancy.

Canadian perinatal health care providers and the patients they serve would benefit from an overarching nation-wide Tdap vaccination strategy and universal vaccine funding to facilitate national implementation of the NACI and SOGC recommendations. Elements of this coordinated approach 
should include efficient clinical training, high-quality patient information materials, and universal coverage and patient access. Lessons learned from the Canadian Tdap vaccination program may be pertinent not only to the Tdap vaccine but also to the implementation of vaccination programs in pregnancy and other health care initiatives more broadly.

\section{References}

1. Abu-Raya B, Bettinger JA, Vanderkooi OG, et al.; Members of the Canadian Immunization Monitoring Program, Active (IMPACT). Burden of children hospitalized with pertussis in Canada in the acellular pertussis vaccine era, 1999-2015. 7 Pediatric Infect Dis Soc 2018 Dec. 8 [Epub ahead of print]. doi: $10.1093 /$ jpids/piy128.

2. NACI update on immunization in pregnancy with Tdap vaccine - 2018. Ottawa: Public Health Agency of Canada; 2018. Available: www.canada.ca/en/public-health/ services/reports-publications/canada-communicable-disease-report-ccdr/monthly -issue/2018-44/issue-3-4-march-1-2018/article-5-update-immunization-pregnancy -vaccine-2018.html (accessed 2020 Apr. 1)

3. Abu Raya B, Edwards KM, Scheifele DW, et al. Pertussis and influenza immunisation during pregnancy: a landscape review. Lancet Infect Dis 2017;17: e209-22.

4. Castillo E, Poliquin V. No. 357 - immunization in pregnancy. 7 Obstet Gynaecol Can 2018;40:478-89.

5. Provincial and territorial routine vaccination programs for healthy, previously immunized adults. Ottawa: Public Health Agency of Canada; 2017. Available: www.canada.ca/en/public-health/services/provincial-territorial-immunization -information/routine-vaccination-healthy-previously-immunized-adult.html (accessed 2020 Apr. 1)

6. Jones CE, Calvert A, Le Doare K. Vaccination in pregnancy - recent developments [published erratum in Pediatr Infect Dis 7 2018;37:465]. Pediatr Infect Dis 7 2018;37:191-3.

7. Tong A, Biringer A, Ofner-Agostini M, et al. A cross-sectional study of maternity care providers' and women's knowledge, attitudes, and behaviours towards influenza vaccination during pregnancy. 7 Obstet Gynaecol Can 2008;30:404-10.

8. Brousseau N, Gagnon D, Vivion M, et al. Expected challenges of implementing universal pertussis vaccination during pregnancy in Quebec: a crosssectional survey. CMA7 Open 2018;6:E391-7.

9. Pertussis immunization in pregnancy in Ontario. Toronto: Public Health Ontario; 2018. Available: www.publichealthontario.ca/en/eRepository/pertussis -immunization-pregnancy.pdf (accessed 2019 Jan. 12).

10. MacDougall DM, Halperin SA. Improving rates of maternal immunization: challenges and opportunities. Hum Vaccin Immunother 2016;12:857-65.

11. Gkentzi D, Katsakiori P, Marangos M, et al. Maternal vaccination against pertussis: a systematic review of the recent literature. Arch Dis Child Fetal Neonatal Ed 2017;102:F456-63.

12. Poliquin V, Greyson D, Castillo E. A systematic review of barriers to vaccination during pregnancy in the Canadian context. 7 Obstet Gynaecol Can 2019;41: 1344-55.

13. Dubé E, Gagnon D, Kaminsky K, et al.; Canadian Immunization Network. Vaccination against influenza in pregnancy: a survey of Canadian maternity care providers. 7 Obstet Gynaecol Can 2019;41:479-88.

14. Wilson R, Paterson P, Larson HJ. Strategies to improve maternal vaccination acceptance. BMC Public Health 2019;19:342.

15. Gauld NJ, Braganza CS, Babalola OO, et al. Reasons for use and non-use of the pertussis vaccine during pregnancy: an interview study. 7 Prim Health Care 2016;8:344-50

16. Strassberg ER, Power M, Schulkin J, et al. Patient attitudes toward influenza and tetanus, diphtheria and acellular pertussis vaccination in pregnancy. Vaccine 2018;36:4548-54.

17. Donaldson B, Jain P, Holder BS, et al. What determines uptake of pertussis vaccine in pregnancy? A cross sectional survey in an ethnically diverse population of pregnant women in London. Vaccine 2015;33:5822-8.

18. Maisa A, Milligan S, Quinn A, et al. Vaccination against pertussis and influenza in pregnancy: a qualitative study of barriers and facilitators. Public Health 2018; 162:111-7.

19. Winslade CG, Heffernan CM, Atchison CJ. Experiences and perspectives of mothers of the pertussis vaccination programme in London. Public Health 2017;146:10-4

20. Table 17-10-0009-01: Population estimates, quarterly. Ottawa: Statistics Canada. Available: https://doi.org/10.25318/1710000901-eng (accessed 2020 Apr. 1).

21. Dubé E, Vivion M, Sauvageau C, et al. How do midwives and physicians discuss childhood vaccination with parents? 7 Clin Med 2013;2:242-59.

22. Greyson D. Information triangulation: a complex and agentic everyday information practice. 7 Assoc Inf Sci Technol 2018;69:869-78.

23. Dubé E, Laberge C, Guay M, et al. Vaccine hesitancy: an overview. Hum Vaccin Immunother 2013;9:1763-73.
24. Mijovic H, Greyson D, Dubé E. More personalized care: pediatric vaccine hesitancy and the patient-provider relationship [abstract]. Int 7 Qual Methods 2018; 17. doi: $10.1177 / 1609406917748701$

25. Canadian Institutes of Health Research, Natural Sciences and Engineering Research Council of Canada, and Social Sciences and Humanities Research Council. Chapter 10: Qualitative research. In: Tri-Council Policy Statement: Ethical Conduct for Research Involving Humans - TCPS 2 (2018). Ottawa: Secretariat on Responsible Conduct of Research; 2018. Available: www.pre.ethics.gc.ca/ eng/tcps2-eptc2_2018_chapter10-chapitre10.html (accessed 2019 Aug. 20).

26. Miles MB, Huberman M, Saldana J. Qualitative data analysis. 3rd ed. Thousand Oaks (CA): Sage Publications; 2013.

27. Thorne S. Interpretive description: qualitative research for applied practice. $2 \mathrm{nd}$ ed. New York: Routledge; 2016.

28. MacDougall DM, Halperin BA, Langley JM, et al. Knowledge, attitudes, beliefs, and behaviors of pregnant women approached to participate in a Tdap maternal immunization randomized, controlled trial. Hum Vaccin Immunother 2016;12:879-85.

29. The Canadian midwifery model of care position statement. Montréal: Canadian Association of Midwives; 2015. Available: https://canadianmidwives.org/wp -content/uploads/2016/06/CAM-MoCPSFINAL-OCT2015-ENG-FINAL.pdf (accessed 2020 Apr. 3)

30. Kaufman J, Attwell K, Hauck Y, et al. Vaccine discussions in pregnancy: interviews with midwives to inform design of an intervention to promote uptake of maternal and childhood vaccines. Hum Vaccin Immunother 2019;15:2534-43.

31. Mohammed H, McMillan M, Roberts CT, et al. A systematic review of interventions to improve uptake of pertussis vaccination in pregnancy. PLoS One 2019;14:e0214538.

32. Paterson P, Meurice F, Stanberry LR, et al. Vaccine hesitancy and healthcare providers. Vaccine 2016;34:6700-6.

33. Turner $\mathrm{N}$. The challenge of improving immunization coverage: the New Zealand example. Expert Rev Vaccines 2012;11:9-11.

34. Potts A, Sinka K, Love J, et al. High uptake of HPV immunisation in Scotland — perspectives on maximising uptake. Euro Surveill 2013;18:20593.

35. Vaccination in pregnancy. Ottawa: Society of Obstetricians and Gynecologists of Canada. Available: www.sogc.org/en/rise/Events/event-display.aspx? EventKey=VACCINE (accessed 2019 Nov. 4).

Affiliations: Vaccine Evaluation Center (Mijović, Greyson, Gemmell, Bettinger), BC Children's Hospital Research Institute, University of British Columbia; Department of Pediatrics (Mijović, Bettinger), Faculty of Medicine, University of British Columbia, Vancouver, BC; Department of Communication (Greyson), University of Massachusetts, Amherst, Mass.; Quebec National Institute of Public Health (Trottier, Vivion, Dubé), Québec, Que.; Department of Pediatrics (Graham), Faculty of Medicine, Dalhousie University, Halifax, NS; Department of Anthropology (Dubé), Université Laval, Québec, Que.

Contributors: Hana Mijović and Devon Greyson contributed equally as first authors. Janice Graham, Ève Dubé and Julie Bettinger supervised the work. Devon Greyson, Janice Graham, Ève Dubé and Julie Bettinger conceived of the study. Devon Greyson and Julie Bettinger designed the study. Devon Greyson, Emily Gemmell, Hana Mijović, Marie-Ève Trottier and Maryline Vivion acquired the data. Hana Mijović drafted the manuscript. All of the authors interpreted the data, revised the manuscript critically for important intellectual content, approved the final version to be published and agreed to be accountable for all aspects of the work.

Funding: This work was supported by an operating grant from the Canadian Immunization Research Network and council grant CIHR IMM-151 599 from the Canadian Institutes of Health Research. Hana Mijović received salary support through the Clinician Investigator Program, University of British Columbia.

Data sharing: Owing to the identifiability of participants and the confidentiality assurances given in the consent form, demographic data and interview transcripts collected during the study cannot be shared publicly. Those with questions about the data should contact the corresponding author, Julie Bettinger, jbettinger@bcchr.ubc.ca.

Acknowledgement: Clara Rubincam collected the data in some of the English-language interviews.

Supplemental information: For reviewer comments and the original submission of this manuscript, please see www.cmajopen.ca/content/8/2/ E377/suppl/DC1. 